\title{
Extract of Helicobacter pylori Ameliorates Parameters of Airway Inflammation and Goblet Cell Hyperplasia following Repeated Allergen Exposure
}

\author{
Yolanda van Wijck ${ }^{\mathrm{a}}$ Gerrit John-Schuster ${ }^{\mathrm{a}}$ Annemarie van Schadewijk ${ }^{\mathrm{a}}$ \\ Ruben L. van den Oever ${ }^{a} \quad K^{2}$ atja Obieglo ${ }^{b}$ Pieter S. Hiemstra ${ }^{a}$ Anne Müller ${ }^{c}$ \\ Hermelijn H. Smits ${ }^{b}$ Christian Taube ${ }^{a}$ \\ a Department of Pulmonology, Leiden University Medical Center, Leiden, The Netherlands; ${ }^{b}$ Department of \\ Parasitology, Leiden University Medical Center, Leiden, The Netherlands; ' Institute of Molecular Cancer Research, \\ University of Zurich, Zurich, Switzerland
}

\section{Keywords}

Asthma Allergic airway inflammation $\cdot$ Helicobacter pylori . Immunomodulation

\begin{abstract}
Background: An inverse relation between Helicobacter pylori infection and asthma has been shown in epidemiological studies. Infection with $\mathrm{H}$. pylori, or application of an extract of it before or after sensitization, inhibits allergic airway disease in mice. Objectives: The aim of this study was to investigate the effect of an extract of $H$. pylori on allergic airway disease induced by repeated allergen exposure in mice that were sensitized and challenged prior to extract application. Method: C57BL/6 mice were intranasally (i.n.) sensitized and challenged with house dust mite (HDM). After a minimum of 4 weeks, mice received the $H$. pylori extract intraperitoneally and were rechallenged i.n. with HDM. Allergen-specific antibodies were measured by ELISA. Cells present in the bronchoalveolar lavage fluid and dendritic cell (DC) subsets in the lung tissue were analyzed by flow cytometry. Tissue inflammation and goblet cell hyperplasia were assessed by histology. Cells of the mediastinal lymph node $(\mathrm{mLN})$ were isolated and in vitro restimulated with HDM or $\mathrm{H}$. pylori extract.
\end{abstract}

Results: Treatment with H. pylori extract before rechallenge reduced allergen-specific IgE, the DC numbers in the tissue, and goblet cell hyperplasia. Cells isolated from $\mathrm{mLN}$ of mice treated with the extract produced significantly more IL-10 and IL-17 after in vitro restimulation with HDM. mLN cells of H. pylori-treated mice that were re-exposed to the H. pylori extract produced significantly more interferon gamma. Conclusions: An extract of $H$. pylori is effective in reducing mucus production and various features of inflammation in HDM rechallenged mice.

(C) 2019 The Author(s)

Published by S. Karger AG, Basel

\section{Introduction}

Asthma is a common syndrome of increasing prevalence [1]. Current treatment of patients with asthma consists of anti-inflammatory drugs such as inhaled corticosteroids, often in combination with bronchodilators. All available treatment options are symptomatic, and symptoms usually return when treatment is paused or stopped. Also, disease-modifying effects by novel treatments with

Edited by: H.-U. Simon, Bern.

Yolanda van Wijck, MSc

Department of Pulmonology, Leiden University Medical Center

Internal Postcode C1-3, PO Box 9600

NL-2300 RC Leiden (The Netherlands)

E-Mail y.van_wijck@lumc.nl 
monoclonal antibodies against IgE $[2,3]$ or IL-5 [4] in patients with severe asthma have not been shown. Furthermore, $5-10 \%$ of patients do not respond to high-dose therapies (a combination of high-dose inhaled/oral corticosteroids and $\beta 2$-adrenoceptor agonists) [5] and the number of novel drugs for asthma that have reached the market is small considering the efforts made and costs accrued [6]. Therefore, further efforts are needed to develop treatments that modify the course of the disease.

Colonization of the stomach with the bacterium $H$. $p y$ lori has been shown to protect against the development of asthma in many epidemiological studies [7, 8]. Experimental infection with Helicobacter pylori or treatment with an $H$. pylori extract before sensitization to allergens inhibited allergic airway inflammation (AAI) in murine models [9-11]. Protection was dependent on the induction of tolerogenic dendritic cells (DCs). We recently showed that AAI was also reduced when the extract was applied at a later point of induction of AAI, i.e., in mice that had been sensitized (but not yet challenged) prior to treatment with the H. pylori extract [12]. Antigen processing by DCs was reduced in vivo and in vitro after exposure to the extract.

However, the model of acute airway disease that we have investigated so far has the drawback that treatment is administered before airway inflammation has developed. In patients with allergic asthma, symptoms and airway inflammation repeatedly occur after allergen exposure [1]. Therefore, we set out to test a potential treatment in a model with repeated allergen exposures to assess the potential therapeutic effect.

We hypothesized that an extract of $H$. pylori would reduce allergic airway disease induced by repeated allergen exposure when applied to mice that were previously sensitized and challenged. To this end, mice were sensitized and challenged prior to a period of rest, treated with the extract, and then rechallenged with the allergen.

\section{Materials and Methods}

\section{Animals}

Eight- to twelve-week-old female C57BL/6 mice were purchased from Charles River ('s-Hertogenbosch, The Netherlands). The animals had free access to food and water, and were housed in individually ventilated cages under specific pathogen-free conditions. Experiments were performed at the animal facilities of the Leiden University Medical Center in Leiden, The Netherlands. The Ethics Committee for Animal Experimentation of the University of Leiden approved the experiments (Dieren Experimentele Commissie, DEC, 12246), which were conducted under strict governmental and international guidelines in accordance with EU Directive 2010/63/EU.
Experimental Protocols for AAI Induced by Rechallenge and Intervention

To induce AAI, mice were intranasally (i.n.) sensitized with $1 \mu \mathrm{g}$ of house dust mite (HDM; Greer, Lenoir, NC, USA) in $50 \mu \mathrm{L}$ of PBS, and challenged by five i.n. applications of $10 \mu \mathrm{g}$ HDM in $50 \mu \mathrm{L}$ of PBS 1 week later (Fig. 1). After a period of rest of at least 4 weeks, the mice were rechallenged by three i.n. applications of 10 $\mu \mathrm{g}$ HDM in $50 \mu \mathrm{L}$ of PBS. As a control, some mice that were sensitized and challenged with HDM received PBS at the time that the other mice were rechallenged with HDM. All i.n. administrations were performed under isoflurane anesthesia $(3 \%, 0.8 \mathrm{~L} / \mathrm{min}) . H$. pylori extract $(200 \mu \mathrm{g})$ in $100 \mu \mathrm{L}$ of PBS was applied intraperitoneally (i.p.) 5 and 3 days before the rechallenge and was continued daily from 1 day before the rechallenge to 1 day thereafter. As a control, the remaining mice received $100 \mu \mathrm{L}$ of PBS i.p. On days in which the mice were both treated with the extract and were rechallenged with HDM, the extract was administered $1 \mathrm{~h}$ before HDM exposure. Mice were analyzed 2 days after the final HDM exposure. Mice were euthanized with an overdose of sodium pentobarbital (Euthasol, AST Farma, Oudewater, The Netherlands). Analyses of the blood, bronchoalveolar lavage (BAL) fluid, and lung tissue were performed as previously published [12]. Blood was collected from the vena cava. After three lavages of the lungs with $1 \mathrm{~mL}$ of PBS that was applied via a tracheal canula, the lungs were perfused with PBS via the heart. The mediastinal lymph node $(\mathrm{mLN})$ was removed and collected in PBS. For flow cytometry analysis, the left lobe of the lungs was collected in PBS. To study the histology, the right lobes were inflated with $3.9 \%(\mathrm{v} / \mathrm{v})$ formaldehyde (Sigma-Aldrich) in PBS after ligature.

\section{Preparation of $\mathrm{H}$. pylori Extract}

The H. pylori extract was prepared as previously published [12]. In short, the H. pylori pre-mouse SS1 strain (PMSS1) was cultured on solid agar plates (bioMérieux Benelux B.V., Zaltbommel, The Netherlands) under microaerophilic conditions $\left(6 \% \mathrm{O}_{2}, 7.1 \%\right.$ $\mathrm{CO}_{2}, 7.1 \% \mathrm{H}_{2}$, and $79.7 \% \mathrm{~N}_{2}$; Anoxomat, Mart Microbiology, Drachten, The Netherlands). After testing of the production of a specific combination of enzymes to assure that the culture contained $H$. pylori, the bacteria were transferred to BBL broth with $10 \%$ FCS (FBS, Gibco, Thermo Fisher Scientific, Waltham, MA, USA) and were further cultured under microaerophilic conditions at $37^{\circ} \mathrm{C}$ in a shaking incubator (New Brunswick Scientific Europe B.V., Nijmegen, The Netherlands) at $185 \mathrm{rpm}$. After checking the purity by Gram-staining of the pellets, the bacteria were pooled, washed in PBS, freeze-thawed in nitrogen, and subjected to a pressure of 3,000 bar in a French pressure cell press (Stansted Fluid Power, Cell Pressure Homogenizer, Harlow, UK). The supernatant was filtered through a $0.2-\mu \mathrm{m}$ filter and the protein content was determined using bicinchoninic acid (BCA protein Kit Pierce, Thermo Fisher Scientific). The concentration of the extract was adjusted using PBS.

\section{Anti-HDM IgE and IgGI}

Serum was obtained by centrifugation of the collected blood, and was diluted in $1 \%$ (w/v) BSA/PBS (Sigma-Aldrich). NUNC Maxisorb plates (Sigma-Aldrich) were coated with $50 \mu \mathrm{g} / \mathrm{mL}$ of $\mathrm{HDM}$ in $0.1 \mathrm{M}$ sodium-carbonate buffer $\mathrm{pH} 9.6$ and non-specific binding sites were blocked with $1 \%$ BSA/PBS. After incubation with the samples, HDM-specific antibodies were detected using biotin-labelled anti-mouse IgE (clone 23G3; Southern Biotech, 


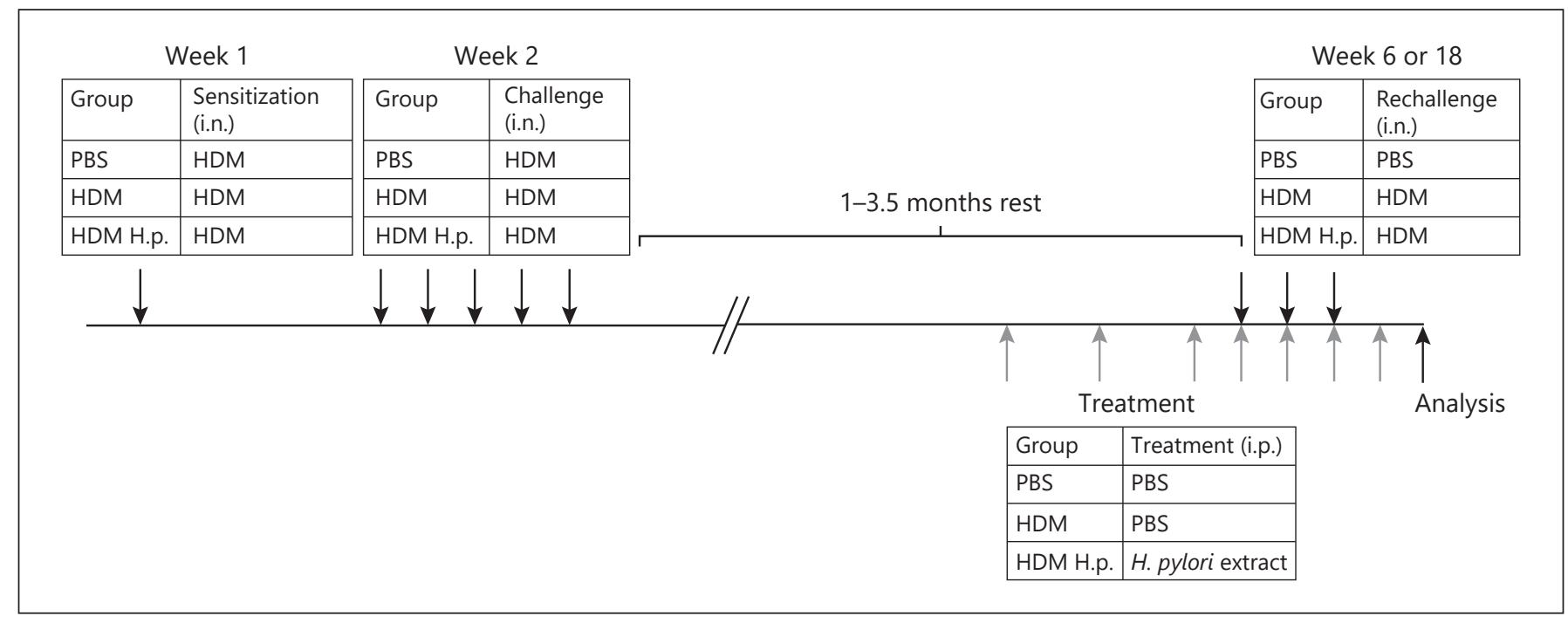

Fig. 1. Experimental setup of the rechallenge model of AAI. C57BL/6 mice were sensitized by i.n. application of HDM, and challenged i.n. with HDM on days 7-11. After 1-3.5 months of rest, the mice were rechallenged with HDM i.n. An extract of $H$. pylori was administered i.p. 5 and 3 days before the rechallenge,

Birmingham, AL, USA) or biotin-labelled anti-mouse IgG1 (clone A85-1; BD Pharmingen, Becton Dickinson B.V., Breda, The Netherlands). Streptavidin coupled to HRP (Sanquin, Amsterdam, The Netherlands) and TMB (3,3',5,5'-tetramethylbenzidine) were added to quantify the antibody binding. A Bio-Rad iMark microplate reader was used for measurement of the ODs at $450 \mathrm{~nm}$.

Flow Cytometry

Quantification of cells in the BAL fluid was performed using Türk's solution (Merck, Schiphol-Rijk, The Netherlands). A single cell suspension was obtained from lung tissue by dissecting the tissue into small pieces. Collagenase/DNAse (Collagenase Type I of Clostridium histolyticum; Calbiochem, San Diego, CA, USA; DNAse I; Sigma-Aldrich) was added for $45 \mathrm{~min}$ at $37^{\circ} \mathrm{C}$. The mixture was filtered through a $70-\mu \mathrm{m}$ cell strainer (BD Falcon, Becton Dickinson). After the lysis of red blood cells, the cells were counted on a Countess automatic cell counter (Invitrogen, Thermo Fisher Scientific). The mLN was filtered through a $70-\mu \mathrm{m}$ cell strainer (BD Falcon, Becton Dickinson) and the single cell suspension was counted using trypan blue. Cells of the BAL fluid, lung tissue, and mLN were labelled with the live/dead stain Aqua (Invitrogen, Thermo Fisher Scientific). Cells of the BAL fluid and lung tissue were fixed with $2 \%$ formaldehyde (Merck) in PBS. The antibodies used for flow cytometry and gating strategies for analysis have been previously published [12]. Treg analysis of cells in the mLN was performed using the eBioscience Foxp3/Transcription factor staining buffer set (Invitrogen). The following antibodies were used: CD4-FITC (GK1.5, eBioscience, Thermo Fisher Scientific), CD25-PerCP-Cy5.5 (PC61, BD Pharmingen, Becton Dickinson B.V.), FOXP3-APC (FJK-16s, eBioscience, Thermo Fisher Scientific), CD3-eFl450 (17A2, eBioscience, Thermo Fisher Scientific). Antibody mixes were supplemented with mouse Fc $\gamma$ RII/III-bind- and daily from 1 day before the rechallenge until 1 day after. The extract was administered $1 \mathrm{~h}$ before HDM exposure on the days of the rechallenge. Analyses were performed 2 days after the final HDM exposure. H.p., H. pylori. ing inhibitor (2.4G2, Bioceros, Utrecht, The Netherlands). A FACSCanto II (BD Bioscience, Becton Dickinson B.V.) and FlowJo (v7.6.5) software (Tree Star, Ashland, OR, USA) were used for data acquisition and analysis. Gates were placed according to unstained and Fluorescence Minus One samples; the gating strategy for the Tregs can be found in online Supplementary Figure 1 (for all online suppl. material, see www.karger.com/doi/10.1159/000500598).

\section{Histology}

Paraffin sections of $4 \mu \mathrm{m}$ were obtained from formaldehydeinflated tissue (fixed for $48 \mathrm{~h}$ at room temperature). Peribronchial inflammation was scored on a 5-point scale (0-4) after staining with hematoxylin and eosin (HE, both Klinipath, Duiven, The Netherlands). In this semiquantitative method, 0 indicates that no inflammation around airways or vessels was detected; 1 indicates that there were some infiltrates of maximum two layers thick; 2 indicates that infiltrates of up to two layers were found at the majority of airways and vessels, and occasionally thicker layers were found; 3 indicates that the majority of airways and vessels were inflamed with more than two layers of cells, but occasionally thinner layers were present; and 4 indicates that all airways and vessels were surrounded by thick layers of inflammatory cells. The analysis was performed by two separate investigators in a blinded fashion. Goblet cell hyperplasia was assessed in tissue stained with Alcian blue and periodic acid-Schiff (PAS) dyes (both from SigmaAldrich). Digital images were taken at $10 \times$ magnification on an Olympus BX41 microscope (Olympus Nederland B.V., Leiderdorp, The Netherlands). Epithelial regions were selected and the fraction of positively stained area corresponding to mucin density was calculated using ImageJ software 1.46r (National Institute of Health, Bethesda, MD, USA). 
Fig. 2. HDM-specific antibodies in serum. a Anti-HDM IgE. b Anti-HDM IgG1. PBS, sensitized and challenged with HDM, rechallenged with PBS; HDM, sensitized and challenged with HDM, rechallenged with HDM; HDM H.p., sensitized and challenged with HDM, treated with $H$. pylori extract, rechallenged with HDM. Results are presented as the mean \pm SEM of two experiments, $n=13-15$ per group. Groups were compared by ANOVA with Dunnett's multiple comparison correction. ns, non-significant. ${ }^{*} p \leq 0.05{ }^{* * * *} p \leq 0.0001$.

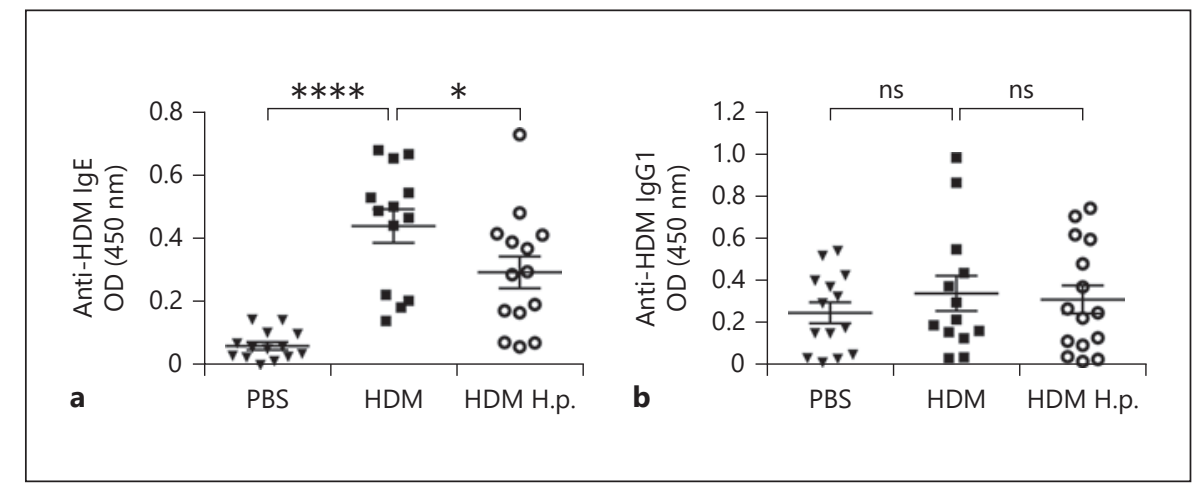

Cytometric Bead Array

The mLN was removed from mice that underwent $H$. pylori or control treatment for AAI. The mLN was mashed through a $70-\mu \mathrm{m}$ falcon cell strainer and the strainer was washed with Dulbecco's PBS (Gibco, Thermo Fisher Scientific). After centrifugation, 200,000 cells were cultured in 10\% FCS RPMI supplemented with $100 \mathrm{U} / \mathrm{mL}$ penicillin and $100 \mathrm{U} / \mathrm{mL}$ streptomycin (Lonza), and stimulated with $(10 \mu \mathrm{g} / \mathrm{mL}) \mathrm{HMD}$ or $(10 \mu \mathrm{g} / \mathrm{mL}) \mathrm{H}$. pylori extract for 4 days. Supernatant was collected and subjected to cytometric bead array (BD Biosciences) according to the manufacturer's protocol.

Statistical Analysis

GraphPad PRISM 7.02 (GraphPad Software Inc., La Jolla, CA, USA) was used to create graphs and to perform statistical analysis. Data are shown as means \pm standard error of the mean (SEM). Differences were considered significantly different at $p$ values $<0.05$ examined by ANOVA and the Dunnett multiple comparisons analysis. For analysis of cytokine production after stimulation of mLN, the two-stage step-up method of Benjamini, Krieger, and Yekutieli was applied to correct for multiple comparisons when performing multiple $t$ tests.

\section{Results}

Mice were initially sensitized with HDM in week 1 and then challenged during week 2 . Following a rest period of at least 4 weeks, the mice were rechallenged with HDM. The mice were i.p. treated with an extract of $H$. pylori prior to a rechallenge (see the experimental set up Fig. 1). HDM-specific IgE was significantly induced following rechallenge with HDM compared to exposure to PBS (Fig. 2a). Treatment with $H$. pylori extract resulted in a significant reduction of anti-HDM IgE levels in serum. However, mice that were rechallenged and treated with the $H$. pylori extract did have significantly higher antiHDM IgE levels compared to mice that received PBS at the moment of rechallenge $(p=0.001)$. No difference in HDM-specific IgG1 levels could be detected between mice rechallenged with HDM compared to mice that received PBS at the rechallenge, and HDM-specific IgG1 was not affected by the treatment (Fig. 2b).

Control mice that received PBS only instead of HDM at rechallenge exhibited low total cell counts and low numbers of eosinophils in BAL fluid, showing that inflammation induced by the first challenge of sensitized mice was mostly resolved after the period of rest (Fig. 3a, b). Rechallenge with HDM led to a significant increase in the number of total cells in the BAL fluid, which was not affected by treatment with $H$. pylori extract. When assessing differential cell counts in BAL fluid, relative and absolute numbers of eosinophils in the BAL fluid were increased following rechallenge with HDM (Fig. 3b, c). Treatment with $H$. pylori extract resulted only in a small, but significant, reduction in the percentage of eosinophils (Fig. 3c). Absolute numbers of eosinophils in BAL fluid were not statistically significantly reduced (Fig. 3b).

In the lung tissue, inflammation was low following i.n. exposure to PBS at the time when other mice were rechallenged with HDM (Fig. 4a, b) as only few infiltrates around the airways and blood vessels of the lung were found. Rechallenge with HDM resulted in a significant increase in inflammation as assessed by HE staining of the lung tissue. Overall inflammation of the lung tissue was not inhibited by treatment with the extract. No mucus production was detected in mice after rechallenge with PBS (Fig. 4a, c), but HDM rechallenge resulted in an increase in mucus production. Mice treated with $H$. pylori extract showed a significant reduction in mucus production. We have reported previously that treatment with the $H$. pylori extract between sensitization and challenge led to a reduction in the number of DCs in the lung, a shift in the subtypes of DCs, and a reduced capacity to process antigen [12]. In the therapeutic model, the number of DCs in the lungs was also reduced, however we did not 


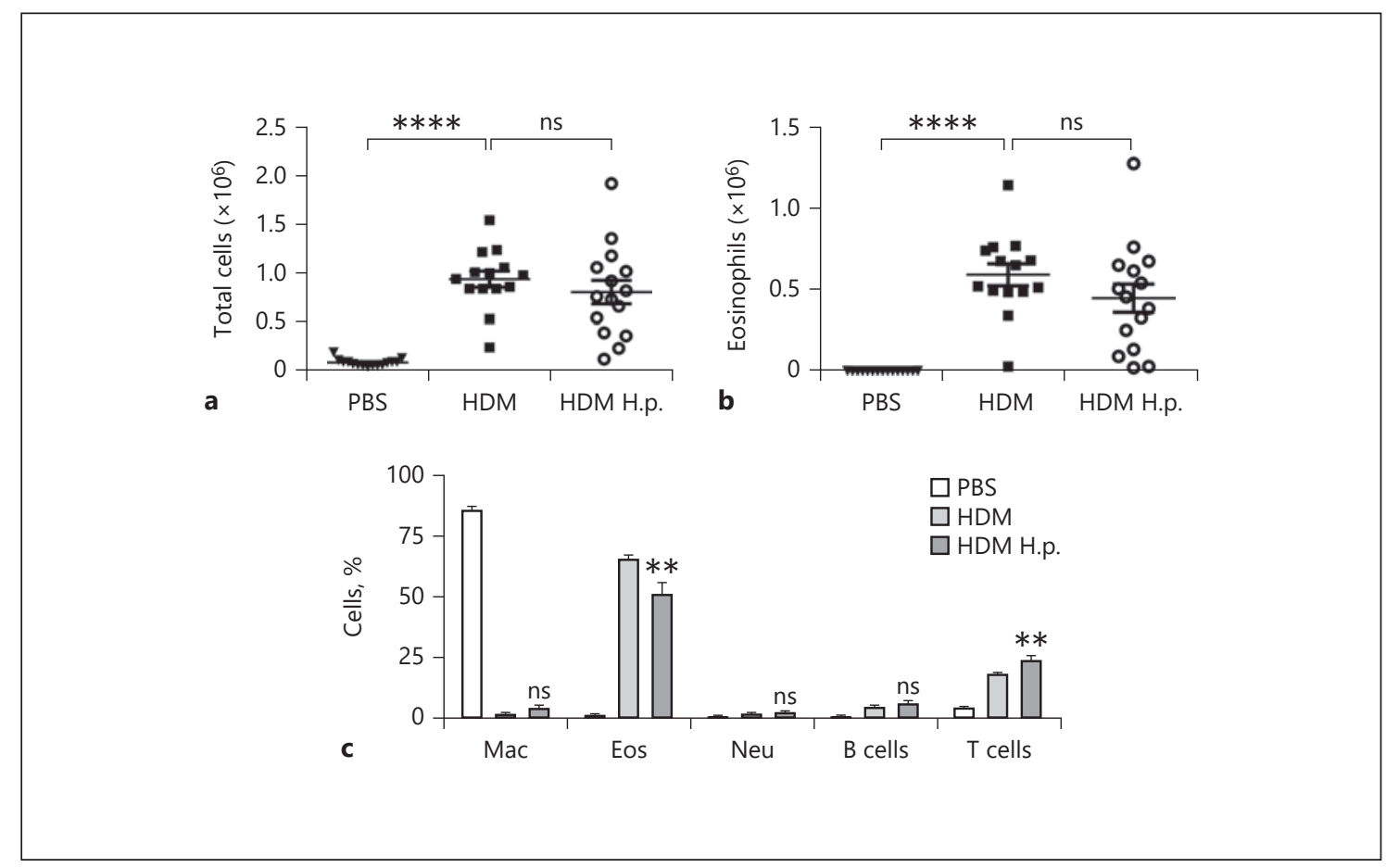

Fig. 3. Analysis of the BAL fluid after treatment of AAI induced by HDM rechallenge with an extract of $H$. pylori. a Exact amount of cells present in the BAL fluid quantified by counting in Türk's solution. b Number of eosinophilic granulocytes present in the BAL fluid. The total amount of cells in the BAL fluid was multiplied by the percentage of eosinophils determined by flow cytometry. c Differential of the BAL fluid, as determined by flow cytometric analysis. Mac, alveolar macrophages; Eos, eosinophils; Neu, neutro-

detect a shift in the ratio of $\mathrm{CD}_{11} \mathrm{~b}^{+}$proinflammatory to $\mathrm{CD}_{103^{+}}$anti-inflammatory DCs, or in the ratio of pro-

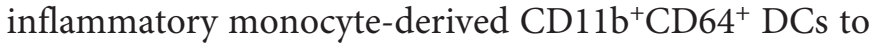
conventional CD11 ${ }^{+} \mathrm{CD} 64^{-}$DCs (Fig. 5).

The mLN is the lymph node that drains the lungs, and it was shown to also be involved in drainage after i.p. injection of ovalbumin in alum [13]. Therefore, we were interested in $\mathrm{mLN}$ responses to the allergen and the extract. When cells of the mLN were restimulated with HDM, the cells of mice that were in vivo treated with $H$. pylori extract prior to the rechallenge produced significantly more IL-10 and IL-17 than cells of mice rechallenged with HDM without being treated with the extract (Fig. 6a). After in vitro restimulation with $H$. pylori extract, cells of mice that were in vivo treated with the extract produced more interferon gamma (IFN $\gamma$ ) than cells of mice that did not receive the extract in vivo. The percentage of Foxp $3^{+}$Tregs in the mLN was decreased after treatment of the mice with $H$. pylori extract (Fig. 6b). phils. PBS, sensitized and challenged with HDM, rechallenged with PBS; HDM, sensitized and challenged with HDM, rechallenged with HDM; HDM H.p., sensitized and challenged with HDM, treated with $H$. pylori extract, rechallenged with HDM. Results are presented as the mean \pm SEM of two experiments, $n=$ 13-16 per group. Groups were compared by ANOVA with Dunnett's multiple comparison correction. ns, non-significant. ${ }^{* *} p \leq$ $0.01 ; * * * p \leq 0.0001$.

\section{Discussion}

Previous studies have shown that the induction of AAI is reduced in mice infected with $H$. pylori or treated with an $H$. pylori extract prior to the induction of allergy [9-11]. Also, the application of an extract of the bacterium after sensitization results in amelioration of the development of the disease [12]. In the present study, we investigated a possible therapeutic effect of treatment directly before a rechallenge in previously sensitized and challenged animals. The results show that intervention at rechallenge leads to a reduction of select, but not all features of the disease. Indeed, HDM-specific IgE in serum was significantly reduced in mice treated with the $H$. pylori extract. In the lung tissue, the number of DCs and extent of goblet cell hyperplasia were significantly reduced. Cells from the mLN of mice treated with the $H$. pylori extract that were restimulated with HDM produced significantly more IL-10 than $\mathrm{mLN}$ cells of mice that were not treated with the extract. 


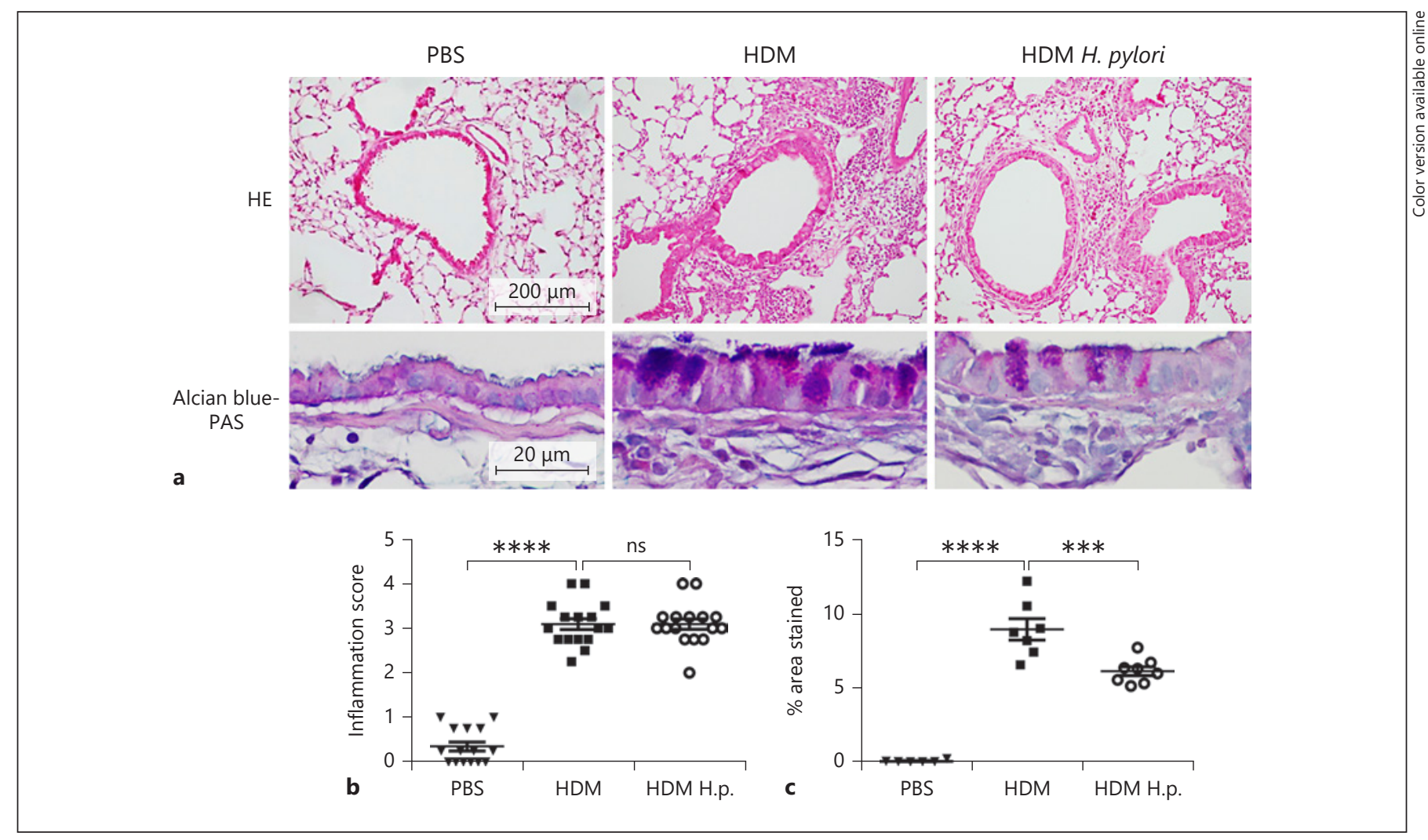

Fig. 4. Lung tissue histology after $H$. pylori extract treatment of AAI induced by HDM rechallenge. a Representative example of peribronchial and perivascular inflammation assessed by $\mathrm{HE}$ staining of lung tissue slides (original magnification $\times 200$ ) and goblet cell hyperplasia by Alcian Blue-PAS staining (original magnification $\times 400)$. b Quantification of inflammation based on $\mathrm{HE}$ staining. c Mucus production assessed by Alcian Blue-PAS staining. PBS, sensitized and challenged with HDM, rechallenged with
PBS; HDM, sensitized and challenged with HDM, rechallenged with HDM; HDM H.p., sensitized and challenged with HDM, treated with $H$. pylori extract, rechallenged with HDM. Results are presented as the mean \pm SEM of two (b) or one (c) experiment(s), $n=15-16$ (b) or $n=6-8$ (c) per group. Groups were compared by ANOVA with Dunnett's multiple comparison correction. ns, nonsignificant. ${ }^{* * *} p \leq 0.001 ;{ }^{* * * *} p \leq 0.0001$.

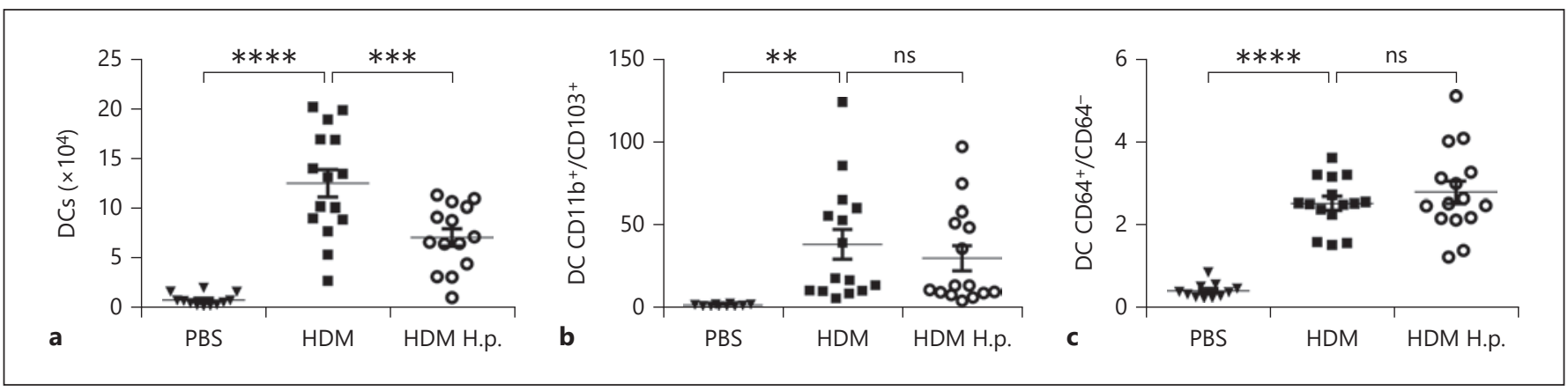

Fig. 5. The DC compartment in lung tissue after $H$. pylori extract treatment of AAI induced by HDM rechallenge. a Quantification of DCs $\left(\mathrm{CD}_{11 \mathrm{c}^{+} \mathrm{MHCII}}{ }^{+}\right)$present in the lung tissue. The total cell count was multiplied by the percentage of DCs assessed by flow

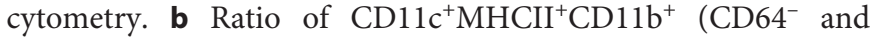
$\mathrm{CD}^{+} 4^{+}$) DCs to $\mathrm{CD} 11 \mathrm{c}^{+} \mathrm{MHCII}^{+} \mathrm{CD} 103^{+} \mathrm{DC}$ in lung tissue, as assessed by flow cytometry. c Ratio of CD $11 \mathrm{c}^{+} \mathrm{MHCII}^{+} \mathrm{CD} 11 \mathrm{~b}^{+} \mathrm{CD} 64^{+}$ DCs to $\mathrm{CD} 11 \mathrm{c}^{+} \mathrm{MHCII}{ }^{+} \mathrm{CD} 11 \mathrm{~b}^{+} \mathrm{CD}^{-} 4^{-} \mathrm{DCs}$ in lung tissue, as as- sessed by flow cytometry. PBS, sensitized and challenged with HDM, rechallenged with PBS; HDM, sensitized and challenged with HDM, rechallenged with HDM; HDM H.p., sensitized and challenged with HDM, treated with $H$. pylori extract, rechallenged with HDM. Results are presented as the mean \pm SEM of two experiments, $n=10-15$ per group. Groups were compared by ANOVA with Dunnett's multiple comparison correction. ns, non-significant. ${ }^{* *} p \leq 0.01$; $^{* * *} p \leq 0.001$; $^{* * *} p \leq 0.0001$. 


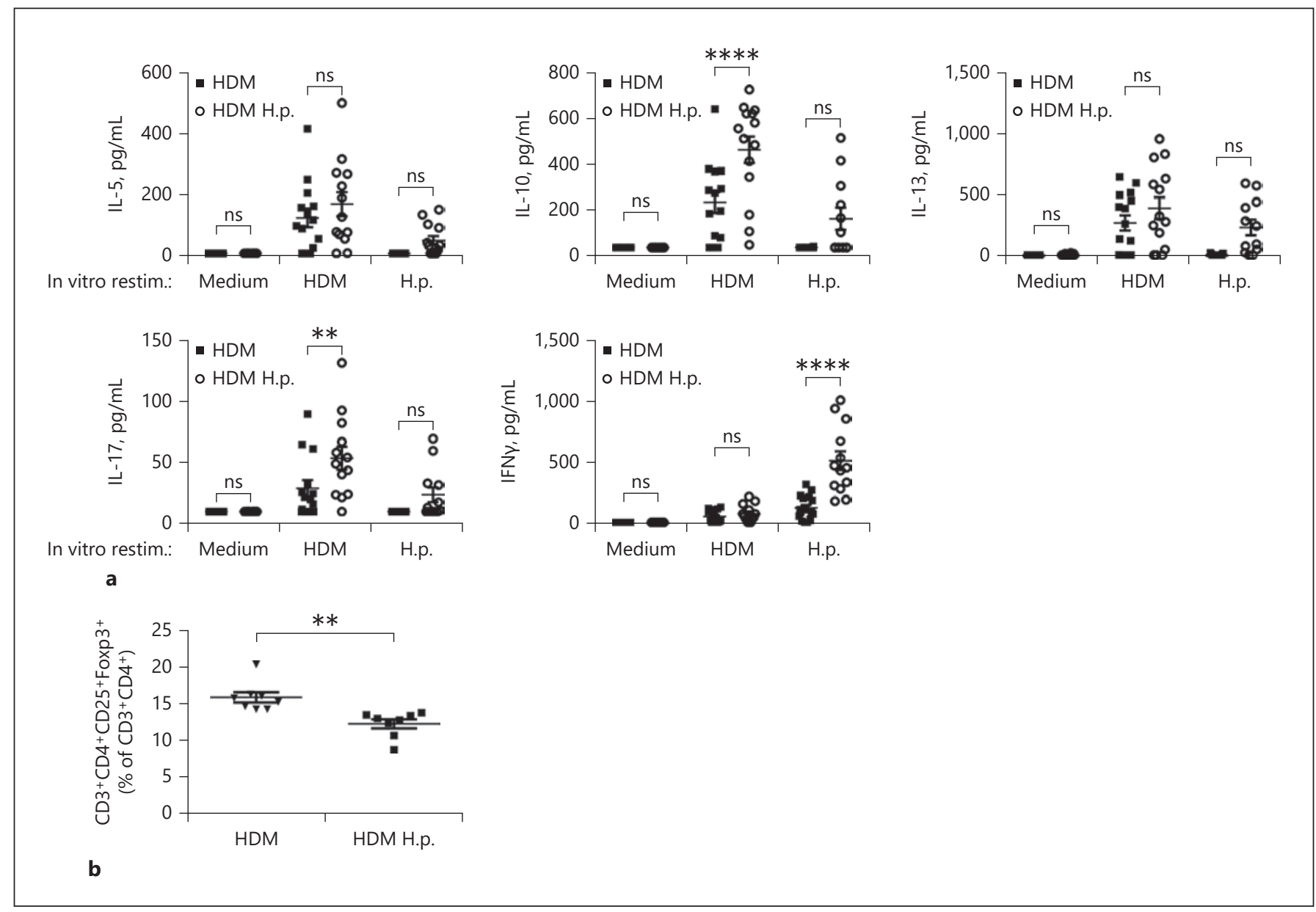

Fig. 6. Effects of in vivo $H$. pylori extract treatment on the mLN. a Cytokine production by cells from the $\mathrm{mLN}$ of $H$. pylori extracttreated mice. Mice were treated with the extract and rechallenged with HDM. The mLN was removed and restimulated with medium, HDM, or H. pylori extract $(10 \mu \mathrm{g} / \mathrm{mL})$ for 4 days. Quantification of IL-5, IL-10, IL-13, IL-17, and IFN $\gamma$ by cytometric bead array. $\mathbf{b}$ Treg in the $\mathrm{mLN}$ of mice after $H$. pylori extract treatment of AAI induced by HDM rechallenge. HDM, sensitized and challenged with HDM, rechallenged with HDM; HDM H.p., sensitized and challenged with HDM, treated with $H$. pylori extract, rechal-

To mimic asthma in vivo, we used a rechallenge model of AAI. This is a relevant model, as patients with allergic asthma can show increased inflammation and symptoms following allergen exposure, but can also be asymptomatic. Still, the residual inflammation in patients that do not experience any symptoms can contribute to a next attack. It was previously shown that inflammation was mostly resolved 6 weeks after sensitization and challenge of mice with OVA, but that a secondary challenge alone induced inflammation in these mice [14], indicating that lenged with HDM. Cytokine results are presented as the mean \pm SEM of two experiments, $n=9-16$ per group. Cytokine production was analyzed by the two-stage step-up method of Benjamini, Krieger, and Yekutieli (multiple $t$ tests, corrected for multiple comparisons). $p$ values are shown only for comparisons where the statistical method showed a discovery. ns, non-significant; restim., restimulation. ${ }^{* *} p \leq 0.01 ;{ }^{* * * *} p \leq 0.0001$. Treg results are presented as the mean \pm SEM of one experiment, $n=8$ per group. ** $p \leq 0.01$. prior allergic airway disease contributes to the development of AAI after a second challenge.

Whereas most attempts to inhibit AAI with micro-organism-based approaches have been described in preventive models, some studies [12,15] showed that AAI can be inhibited when micro-organism preparations are applied to sensitized mice. Fewer studies report on successfully inhibiting AAI when applying micro-organism preparations after a prior sensitization and challenge $[15$, 16]. Thorburn et al. [15] showed that components of 
Streptococcus pneumoniae applied during established disease inhibited allergic airway disease by the induction of Tregs that inhibited natural killer T-cells. Lagranderie et al. [16] demonstrated inhibition of AAI by a freeze-dried preparation of Mycobacterium bovis BCG applied after the induction of established disease, which was associated with the induction of the immunosuppressive cytokine IL-10. The persistence of the observed treatment effects requires further analysis. Navarro et al. [17] showed that a mix of bacterial extracts (Broncho-Vaxom, used for recurrent respiratory infections) could inhibit AAI when applied orally from 1 day before the sensitization until the challenge. When mice were rechallenged 3 weeks later, treatment was no longer effective, indicating that protection was short lived. Others showed that treatment that was started before the sensitization was still effective when mice were challenged 30 days after infection with Escherichia coli [18].

In our experience, treatment at a later stage of the disease (before a second challenge) affected goblet cell hyperplasia, but had less effect on inflammation compared to treatment before the first challenge [12]. To reduce inflammation caused by a rechallenge, more frequent injections with $H$. pylori extract for a longer period of time might be required.

The largest effect of treatment was on goblet cell hyperplasia, total amount of DCs in the lung tissue, antigenspecific IgE in serum, and IL-10 in the mLN. H. pylori extract affects antigen processing by DCs [12]. It is possible that the extract also induced IL-10 production by DCs, or that T cells that are skewed by the DCs produce IL-10. This is supported by the increased production of IL-10 after restimulation with HDM of cells of the mLN in mice treated with the extract. IL-10 has a central role in the control of asthma, and is known to inhibit many different cells and their functions in allergic disease, including mast cells [19-21]. Production of IL-10 may have led to reduced goblet cell hyperplasia, either by an effect on mast cells, or by another mechanism. Another possibility is that goblet cell hyperplasia is reduced as a consequence of reduced IL-13 production. The role of IL-13 in mucus hypersecretion and induction of airway hyperresponsiveness in mice has been well described and blocking IL-13 mainly reduces these features with less effect on airway inflammation [22, 23]. IL-13 is known to induce goblet cell hyperplasia directly in mouse epithelial cells [22]. The role of IL-13 in mucus hypersecretion is furthermore supported by in vitro studies with cultured human airway epithelial cells, showing the ability of IL-13 to induce goblet cell hyperplasia [24, 25]. Two ways that treatment with the extract could lead to reduced IL-13 production is that the extract affects Th2 cell skewing by DCs, or that the reduction in IgE leads to reduced IL-13 release by mast cells [26]. Although we did not observe a reduction in production of Th2 cytokines by cells in the $\mathrm{mLN}$ after restimulation with the allergen in mice treated with the extract, we cannot formally exclude that IL-13 is reduced in BAL fluid or lung tissue. Applying the extract i.n. with the intention to target the epithelium directly proved to be less successful in inhibiting AAI than i.p. injection in the more acute models [Y. van Wijck; unpubl. observations].

In clinical research it has been recognized that different phenotypes in asthma exist, which are susceptible to different treatment approaches. Therefore, modelling asthma also needs to consider various models for different phenotypes [6]. The most widely investigated asthma phenotype in such animal models is allergic, eosinophilic asthma. The current results show that certain characteristics of AAI and airway remodelling can also be inhibited in a more chronic model of the disease by using an extract of $H$. pylori.

To conclude, treatment of AAI with an extract of $H$. pylori at the time of a rechallenge inhibited goblet cell hyperplasia and selected inflammatory features of the disease. Therefore, this extract or its active components might be meaningful as a new therapeutic option for patients with asthma.

\section{Acknowledgement}

Mouse Fc $\gamma$ RII/III-binding inhibitor (2.4G2) was a kind gift of Louis Boon (Bioceros, Utrecht, The Netherlands). The authors would like to thank Anneloes Blok and the Leiden Institute of Chemistry at the Gorlaeus Laboratories of the Leiden University for providing their French Press for preparation of $H$. pylori extracts. Padmini Khedoe, Anne van der Does, and Simone Hermans provided technical assistance.

\section{Statement of Ethics}

Animal experiments conform to internationally accepted standards and have been approved by the appropriate institutional review body (Ethics Committee for Animal Experimentation of the University of Leiden, Dieren Experimentele Commissie, 12246).

\section{Disclosures Statement}

The authors have no conflicts of interest to declare. 


\section{Funding Sources}

This work was supported by Lung Foundation Netherlands (consortium grant 5115015).

\section{Author Contributions}

Y.v.W., G.J-.S., K.O., P.S.H., A.M., H.H.S., and C.T. designed the experiments. Y.v.W., G.J-.S., A.v.S., R.L.v.d.O., and K.O. performed the experiments and analyzed the data. Y.v.W., P.S.H., and C.T. drafted the manuscript. All authors approved the final manuscript.

\section{References}

1 Global Initiative for Asthma. Global strategy for asthma management and prevention. 2018 [cited 2019 Feb]. Available from: www. ginasthma.org.

2 Baena-Cagnani CE, Teijeiro A, Canonica GW. Four-year follow-up in children with moderate/severe uncontrolled asthma after withdrawal of a 1-year omalizumab treatment. Curr Opin Allergy Clin Immunol. 2015 Jun;15(3):267-71.

3 Domingo C, Pomares X, Navarro A, Amengual MJ, Montón C, Sogo A, et al. A stepdown protocol for omalizumab treatment in oral corticosteroid-dependent allergic asthma patients. Br J Clin Pharmacol. 2018 Feb;84(2): $339-48$.

4 Farne HA, Wilson A, Powell C, Bax L, Milan SJ. Anti-IL5 therapies for asthma. Cochrane Database Syst Rev. 2017 Sep;9:CD010834.

5 Bosnjak B, Stelzmueller B, Erb KJ, Epstein MM. Treatment of allergic asthma: modulation of Th2 cells and their responses. Respir Res. 2011 Aug;12(1):114.

6 Mullane K. The increasing challenge of discovering asthma drugs. Biochem Pharmacol. 2011 Sep;82(6):586-99.

7 Taube C, Müller A. The role of Helicobacter pylori infection in the development of allergic asthma. Expert Rev Respir Med. 2012 Aug; 6(4):441-9.

8 Zhou X, Wu J, Zhang G. Association between Helicobacter pylori and asthma: a meta-analysis. Eur J Gastroenterol Hepatol. 2013 Apr; 25(4):460-8.

9 Arnold IC, Dehzad N, Reuter S, Martin H, Becher B, Taube C, et al. Helicobacter pylori infection prevents allergic asthma in mouse models through the induction of regulatory $\mathrm{T}$ cells. J Clin Invest. 2011 Aug;121(8):3088-93.

10 Oertli M, Sundquist M, Hitzler I, Engler DB, Arnold IC, Reuter S, et al. DC-derived IL-18 drives Treg differentiation, murine Helicobacter pylori-specific immune tolerance, and asthma protection. J Clin Invest. 2012 Mar; 122(3):1082-96.
11 Engler DB, Reuter S, van Wijck Y, Urban S, Kyburz A, Maxeiner J, et al. Effective treatment of allergic airway inflammation with Helicobacter pylori immunomodulators requires BATF3-dependent dendritic cells and IL-10. Proc Natl Acad Sci USA. 2014 Aug; 111(32):11810-5.

12 van Wijck Y, de Kleijn S, John-Schuster G, Mertens TC, Hiemstra PS, Müller A, et al. Therapeutic Application of an Extract of Helicobacter pylori Ameliorates the Development of Allergic Airway Disease. J Immunol. 2018 Mar;200(5):1570-9.

13 Kool M, Soullié T, van Nimwegen M, Willart MA, Muskens F, Jung S, et al. Alum adjuvant boosts adaptive immunity by inducing uric acid and activating inflammatory dendritic cells. J Exp Med. 2008 Apr;205(4):869-82.

14 Kanehiro A, Ikemura T, Mäkelä MJ, Lahn M, Joetham A, Dakhama A, et al. Inhibition of phosphodiesterase 4 attenuates airway hyperresponsiveness and airway inflammation in a model of secondary allergen challenge. Am J Respir Crit Care Med. 2001 Jan;163(1):17384.

15 Thorburn AN, Foster PS, Gibson PG, Hansbro PM. Components of Streptococcus pneumoniae suppress allergic airways disease and NKT cells by inducing regulatory T cells. J Immunol. 2012 May;188(9):4611-20.

16 Lagranderie M, Abolhassani M, Vanoirbeek J, Lefort J, Nahori MA, Lapa E Silva JR, et al. Mycobacterium bovis BCG killed by extended freeze-drying reduces airway hyperresponsiveness in 2 animal models. J Allergy Clin Immunol. $2008 \mathrm{Feb} ; 121(2): 471-8$.

17 Navarro S, Cossalter G, Chiavaroli C, Kanda A, Fleury S, Lazzari A, et al. The oral administration of bacterial extracts prevents asthma via the recruitment of regulatory $\mathrm{T}$ cells to the airways. Mucosal Immunol. 2011 Jan;4(1): 53-65.
18 Nembrini C, Sichelstiel A, Kisielow J, Kurrer M, Kopf M, Marsland BJ. Bacterial-induced protection against allergic inflammation through a multicomponent immunoregulatory mechanism. Thorax. 2011 Sep;66(9): 755-63.

19 Hawrylowicz CM. Regulatory T cells and IL10 in allergic inflammation. J Exp Med. 2005 Dec;202(11):1459-63.

20 Hawrylowicz CM, O'Garra A. Potential role of interleukin-10-secreting regulatory $\mathrm{T}$ cells in allergy and asthma. Nat Rev Immunol. 2005 Apr;5(4):271-83.

21 Palomares O, Martín-Fontecha M, Lauener R Traidl-Hoffmann C, Cavkaytar O, Akdis M, et al. Regulatory $\mathrm{T}$ cells and immune regulation of allergic diseases: roles of IL-10 and TGF- $\beta$. Genes Immun. 2014 Dec;15(8):51120.

22 Kuperman DA, Huang X, Koth LL, Chang GH, Dolganov GM, Zhu Z, et al. Direct effects of interleukin-13 on epithelial cells cause airway hyperreactivity and mucus overproduction in asthma. Nat Med. 2002 Aug;8(8):8859.

23 Taube C, Duez C, Cui ZH, Takeda K, Rha YH, Park JW, et al. The role of IL-13 in established allergic airway disease. J Immunol. 2002 Dec; 169(11):6482-9.

24 Kistemaker LE, Hiemstra PS, Bos IS, Bouwman S, van den Berge M, Hylkema MN, et al. Tiotropium attenuates IL-13-induced goblet cell metaplasia of human airway epithelial cells. Thorax. $2015 \mathrm{Jul} ; 70$ (7):668-76.

25 Zuyderduyn S, Ninaber DK, Schrumpf JA, van Sterkenburg MA, Verhoosel RM, Prins FA, et al. IL-4 and IL-13 exposure during mucociliary differentiation of bronchial epithelial cells increases antimicrobial activity and expression of antimicrobial peptides. Respir Res. 2011 May;12(1):59.

26 Burd PR, Thompson WC, Max EE, Mills FC Activated mast cells produce interleukin 13. J Exp Med. 1995 Apr;181(4):1373-80. 